\title{
12. VERZEICHNIS DER ZITIERTEN HANDSCHRIFTEN UND DRUCKE VOR 1600
}

\author{
a) HANDSCHRIFTEN
}

AQUILA, Capestranoarchiv, Carte deteriorate n. 62. Dazu. S. 72

GRAZ, Zentralbibliothek der Wiener Franziskanerprovinz, Cod. A 63/51. Dazu S. 25 A. 60 HAMBURG, Staats- und Universitätsbibliothek, Cod. 8 in scrin. Dazu S. 24 A. 56

LJUBLJANA, Franziskanerkloster, Cod. 6772. Dazu S. 25 A. 60

MELK, Stiftsbibliothek, Cod. 800 (olim 863, P 57). Dazu S. 50, 71

MÜNCHEN, Bayerische Staatsbibliothek, Clm 19651. Dazu S. 71

MÜNCHEN, Universitätsbibliothek, $4^{\circ}$ Cod. ms. 810. Dazu S. 24

PRAG, Nationalbibliothek:

Cod. Osek 71. Dazu S. 22 f.

Cod. XIII H 3b. Dazu S. 23 A. 54

PRAG Strahov, Cod. DG III 15. Dazu S. 22, 23 A. 49

PRESSBURG, Kapitelbibliothek: Verschollener Sammelkodex vor 1425. Vgl. J. Sорко,

Codices latini medii aevi bibliothecarum Slovaciae. Martin 1981, Nr. 61. Dazu S. 35, 78

A. 270

ROM, Biblioteca Apostolica Vaticana:

Cod. Pal. lat. 1787. Dazu S. 15 ff. und passim; Abb. 1-12, 14-18, 24-26

Cod. Pal. lat. 1789. Dazu S. 11, 29 f., 69, 71, 77, 80

Cod. Pal. lat. 1939. Dazu S. 15 A. 21, 29 A. 73

TEPL, Stiftsbibliothek, Cod. 39. Dazu S. 20 ff., 42; Abb. 19-22, 27-30

VENEDIG, Marciana, Cod. Marc. lat. DCLIX. Dazu S. 70

WIEN, Österreichische Nationalbibliothek:

CVP 23*. Dazu S. 42 A. 129, 49, 71

CVP 338. Dazu S. 12

CVP 1775. Dazu S. 22; Abb. 13, 23

CVP 2289. Dazu S. 28 A. 66

CVP 2368. Dazu S. 23, 24 A. 56, 28 A. 66; Abb. $31 \mathrm{f}$.

CVP 2598. Dazu S. $13 \mathrm{f}$.

CVP 2828. Dazu S. 23 A. 49

CVP 2868. Dazu S. 51 ff. und passim; Abb. 39 f.

CVP 2945. Dazu S. $43 \mathrm{ff}$ und passim; Abb. $37 \mathrm{f}$.

CVP $7999^{\circ}$. Dazu S. 52

CVP 9531. Dazu S. 49 A. 141

CVP S. n. 2214. Dazu S. 13 f.

CVP S. n. 2617. Dazu S. 23, 24 A. 56

CVP S. n. 2618. Dazu S. 28 A. 66

CVP S. n. 4643. Dazu S. 13

CVP S. n. 4772. Dazu S. 25 A. 60 


\section{b) Drucke vor 1600}

Introito e Porta compiuto per Meistro Adamo de Roduila, $147 \%$ adi 12 Augusto (Venedig). Wien, Österreichische Nationalbibliothek, Sign. 12 H. 6

Anastatischer Nachdruck mit Einleitung von Alda Bart Rossebastiano (Neolatina rariora I/Lexicalia 1. Turin 1971).

(S)Olenissimo Uochabuolista... Wien, (Stephan Koblinger ?) 1482. Wien, Österreichische Nationalbibliothek, Sign. Ink.12 H 6.

/Catholicum abbreviatum/. (Paris, Antoine Caillaut, wohl 1482). München, Bayerische Staatsbibliothek, Sign. C-195.

/Catholicum abbreviatum/. (Paris, Antoine Caillaut, um 1482-1484). Paris, Bibliothèque Nationale, Sign. Rés. X 1599.

/Catholicum abbreviatum/ (Paris, Antoine Caillot, um 1482-1484). Paris, Bibliothèque Nationale, Sign. Rés. X 1598

Passio Pragensium. Allhie heben sich an die klegelichen geschichte vnde erbermiglichenn die czu Prage vo(n) denn vormaledeyten ketzeren wider die cristen ... (Leipzig, Conrad Kachelofen, 1483). London, British Museum, Sign. IA 11574

Donatus minor....Straßburg, Johann Prüss (nicht nach 1487). Das Wiener Exemplar illuminiert, mit Devise AEIOU auf der Rückseite des letzten Blattes. Wien, Österreichische Nationalbibliothek, Sign. Ink. 21.E.28.

Vocabula pro iuvenibus ... (Leipzig, Matthias Landsberg, o. J.). Vgl. dazu Wizdalková, Nejstarsí známý tistený vokabulár latinsko-česko-nemecký, II 136-137

Vocabula pro iuvenibus ... (Leipzig, Conrad Kachelofen, zwischen 1489 und 1497). Gnesen, Biblioteka Archidiecezjalna, Sign. AA C frg.: fasc. A 8

Vocabula pro iuvenibus... (Leipzig, Conrad Kachelofen, um 1492). Stuttgart, Landesbibliothek, Sign. * an. Inc. $4^{\circ} 1498 \mathrm{HB}$

Vocabula pro iuvenibus ... (Nürnberg, Friedrich Creussner, um 1493-1497). München, Bayerische Staatsbibliothek, Sign. $4^{\circ}$ Inc. c.a. $1361 \mathrm{~d} / 6$

Schedel, Hartmann, Liber Chronicarum Das Buch der Croniken und Geschichten. Nürnberg 1493, Wien, Österreichische Nationalbibliothek, Sign. Ink. 6. A. 15

Clement von Grätz. Dyss puchlein hat gemacht vnnd erfarn Mayster Clement von Gracz von allen paden dye von natur hayss sint ... Brünn 1495. London, British Museum, Sign. IA 51720

Vocabula pro iuvenibus ... (Leipzig, Gregorius Böttiger, um 1495). Breslau, Universitätsbibliothek, Sign. XV. Q. 735

Vocabula rerum variarum iuvenibus non minus necessaria qu(am) utilia. (Leipzig, Gregorius Böttiger, um 1495). Brünn, Universitätsbibliothek, Sign. AP 169, adl. 1

Vocabula pro iuvenibus ... (Augsburg, um 1495) in zwei Varianten. München, Bayerischer Staatsbibliothek, Sign.* $4^{\circ}$ L. lat. 484 bzw. München, Universitätsbibliothek, Sign. * $4^{\circ}$ Inc. lat. $375: 7$

Vocabula pro iuvenibus ... Augsburg, Johann Schawr 1496. München, Bayerische Staatsbibliothek, Sign. * $4^{\circ}$ Inc. c.a. $1067 \mathrm{a} / 1$

Missale Pragense emendatu(m) iuxta rubrica (m) Pragensis ecclesiae. Leipzig, Conrad Kachelofen, 1498. London, British Museum, Sign. IC 12348 
Vocabula pro iuvenibus ... Basel, Michael Furter (um 1499). Kriegsverlust der Berliner Staatsbibliothek, vgl. E. Voulliéme, Die Inkunabeln der Königlichen Bibliothek und der anderen Berliner Sammlungen. Beiheft zum Zentralblatt für Bibliothekswesen XXX. Leipzig 1906, 590 Vocabula pro iuvenibus ... Leipzig, Melchior Lotter 1499. Paris, Bibliothèque Mazarine, Sign. Inc. 556 (3)

Vocabula pro iuvenibus ... Leipzig, Melchior Lotter, 1500. Krakau, Biblioteka Jagiellonska, Sign. Inc. 2904

Vocabula juvenibus multum necessaria ... Lübeck, Steffen Arndes, 1500. Göttingen, Universitätsbibliothek, Sign. * 8 Ling. IV 3770

Vocabula pro iuvenibus ... (Augsburg, Johann Froschauer, um 1505). München, Bayerische Staatsbibliothek, Sign. * $4^{\circ}$ Inc. s. a. 1958. Bei Franz Claes, Bibliographisches Verzeichnis der deutschen Vokabulare und Wörterbücher, gedruckt bis 1600. Hildesheim-New York 1977, noch die Varianten Nr. 171 und 172.

Vocabula pro iuvenibus ... Nürnberg, Hieronymus Hoeltzel, 1508. München, Bayerische Staatsbibliothek, Sign. * $4^{\circ}$ L. lat. 486

Pauli Crosnensis Rutheni Artium Liberalium magistri.Poetaeque qua $(m)$ suauisissimi Panegyrici ad diuu(m) Ladislau $(m)$ Pannoniae regem uictoriosissimu $(m)$ : \& praesule $(m): \&$ $\operatorname{sanctu}(m)$ Stanislau $(m)$ praesule $(m)$ : ac martyre $(m)$ Poloniae pleraque alia connexa carmina ... Wien, Johann Winterburger, 1509. Wien, Österreichische Nationalbibliothek, Sign. + 38. R. 52

Roderici Dubravi Bohemi Libellus de componendis Epistolis ... Attamen hunc nostrum relegens studiose libellum Dispeream: doctum ni lepidumque voces... Wien, Hieronymus Vietor, 1511. Wien, Österreichische Nationalbibliothek, Sign. + 44. G. 28

Johann Aquensis, Vocabularius, cuius nomen Lactifer. Pilsen, Nikolaus Bakalarz 1511. Wien, Österreichische Nationalbibliothek, Sign. 126.024-B

Lateinisch-tschechisch-deutsches Vokabularfragment, vor 1513. Prag, Strahovbibliothek, Sign.554 / zl.

Dictionarium: trium linguarum. Latine. Teutonice: Boemice potiora vocabula continens: peregrinantibus apprime vtilis... Wien, Hieronymus Vietor \& Johann Singriener, 1513. Wien, Österreichische Nationalbibliothek, Sign. 20.518-B

Vocabularz Latine e Cescy a Niemetzky kaz dému mlademu y Staremu welmi potrzebny a vzyteeny. Vocabularius rerum. (Nürnberg) Jodocus Gutknecht (nach 1513). Zwickau, Ratsschulbibliothek, Sign. 15. 10. 44/6

Vocabularius hic cuiq(ue) perutilis p(rae) sertim tamen cupienti; aut linguam disertissimam ac ornatissimam Boemicam: aut multas per regiones diffusam Theutonica $(m)$ scire .... Pressus est in proclamato Lipsensi Oppido a Vulffgange Monacen(sis)... 1514. Prag, Universitätsbibliothek, Sign. 54 S 573

Vocabularium Latinis, Gallicis et Theutonicis verbis scriptum ... Lyon, Jehan Thomas 1514. München, Bayerische Staatsbibliothek, Sign. * Polyglot. 86

Joannes Murmellius, Dictionarius variarum rerum, tum pueris tum adultis utilissimus, cum Germanica atque Polonica interpretatione. Krakau, Hieronymus Vietor (1526). Krakau, Biblioteka Jagiellonska. Anastatischer Nachdruck mit Einführung von Wlodzimierz Gruszczynski in Biblioteka tradicji literackych 13B, Krakau 1997. 
(Franciscus Mymerus), Dictionarium trium linguarum, Latinae, Teutonicae ac Polonicae ... Krakau, Matthias Scharfenberg, 1528. Uppsala, Universitätsbibliothek, Sign. Obr. 53 : 360. Anastatischer Nachdruck mit Einführung von Wlodzimierz Gruszczynski in Biblioteka tradycji literackych 13A, Krakau 1997.

Quinqve Lingvarvm vtilissimvs Vocabularius: Latinae, Italicae, Gallicae, Bohemicae \& Alemanicae, valde necessarius per mundum versari cupientibus ... Nürnberg, Friedrich Peypus, 1531. Wien, Österreichische Nationalbibliothek, Sign. 46. S. 35

Nauczenie kratke obogi řečij Niemecke o Čzeske včitigse čijsti y mluwiti Čzechom niemeckij a Niemcom česky. Eyn kurcze vnderweisung beyder sprach Deutsch und Behemisch zulernen lesen und reden Den Behemen deutsch vnd den deutschen behemisch. Pilsen, Johann Peck 1531. Prag, Nationalmuseum, Sign. 26. G. 8, Adl. 2

Fragment (Elementa latinae, bohemicae ac germanicae linguae $=$ Nachdruck des Dictionarius trium linguarum). (Pilsen, Johann Peck, 1532). Prag, Nationalmuseum, Sign. 26. G. 8, Adl. 3

Gabriel Pesti. - Nomenclatura sex lingvarum Latinae, Italicae, Gallicae, Bohemicae, Hungaricae \& Germanicae Lingua autem Hungarica nouiter accessit, cum Latinarum dictionumque plurimarum, quae prius deprauatae fuerant restitutione. Per Gabrielem Pannonium Pesthinum.... Wien, Johann Singriener, 1538. Wien, Österreichische Nationalbibliothek, Mikrofilm Nr. 731

Lexikon symphonon Sigismundi Gelenii iam duplo auctius ....Basel, Robert Winter, 1544. Wien, Österreichische Nationalbibliothek, Sign. 73. V. 64

Gabriel Pesti. - Nomenclatura sex lingvarum ... (Wien, Johann Singriener jr. 1554). Res Litteraria Hungariae Nr. 103

Gabriel Pesti. - Nomenclatura sex lingvarum ...Fleissig corrigirt vnd gepessert. Wien, Johann Singriener jr. 1559. Wien, Österreichischen Nationalbibliothek, Sign. 290.396-C (Kopie)

Gabriel Pesti. - Nomenclatura sex lingvarum ...Fleissig corrigirt vnd gepessert. Wien, Johann Singriener jr. (1558-)1561. Res Litteraria Hungariae Nr. 166

Libellus elegans Galeoti Martii de egregie, sapienter, iocose dictis ac factis Mathiae Vngariae regis ad inclytum ducem Joannem ejus filium ...Wien, Zimmermann, 1563. Wien, Österreichische Nationalbibliothek, Sign. CP. 1. E. 5

Gabriel Pesti. - Nomenclatura sex lingvarum ... Wien, Philipp Püdninger 1568. Wien, Österreichische Nationalbibliothek, Sign. 304.220-A. Alt Rara

Gabriel Pesti. - Nomenclatura sex lingvarum ... Wien, Caspar Stainhofer, 1568. München, Bayerische Staatsbibliothek, Sign. * Polygl. 110

Variorum rerum vocabula cum sueca interpretatione. Formulae puerilium colloquiorum. Stockholm 1579. Faksimile av de enda kända exemplaren i Preussische Staatsbibliothek, Berlin med en efterskrift av Isak Collijn (Faksimileupplagor av äldre svenska tryck. VI) Stockholm 1925

Megiser, Hieronymus, Dictionarivm quatvor linguarvm, videlicet, Germanicae, Latinae, Illyricae (quae vulgo Sclavonica appellatur) \& Italicae, sive Hetruscae. Graecii Styriae a Johanne Fabro 1592, Österreichische Nationalbibliothek, Sign. 73. M. 173 